\title{
Evaluación de la toxicidad de mezclas binarias de cobre, cinc y arsénico sobre Daphnia obtusa (Kurz, 1874) (Cladocera, Crustacea)
}

\author{
Hernán Gaete * y Claudia Chávez
}

Departamento de Biología y Ciencias Ambientales, Centro de Investigación y Gestión de Recursos Naturales CIGREN, Facultad de Ciencias, Universidad de Valparaíso. Av. Gran Bretaña 1111, Playa Ancha, Valparaíso, Chile

* Corresponding author: hernan.gaete@uv.cl

Recibido: 10/4/07

Aceptado: 30/7/07

\begin{abstract}
Evaluation of the toxicity of binary mixtures of copper, zinc, and arsenic on Daphnia obtusa (Kurz, 1874) (Cladocera, Crustacea)

The protection of aquatic ecosystems is based on environmental norms that consider the maximum concentration allowed for individual chemical substances. Nevertheless, in reality there are complex mixtures in which multiple interactions among the constituents occur (i.e. additive, synergic and antagonistic). Among the chemical agents that are present in high concentrations in the aquatic ecosystems affected by mining activity are copper, zinc, and arsenic. The aim of this investigation was to determine what type of interactions occur between these elements and therefore, cute toxicity tests between binary mixtures of $\mathrm{Zn}: \mathrm{As}, \mathrm{Zn}: \mathrm{Cu}$ and $\mathrm{Cu}$ :As on Daphnia obtusa were performed. The type of interaction was determined through the concentration-addition and synergistic ratio models. The results show that zinc exerts an antagonistic interaction on arsenic, while arsenic has a synergistic effect on zinc. As for the interaction $\mathrm{Zn}$ : $\mathrm{Cu}$, when the concentration of zinc in the mixture was increased, an antagonistic effect on copper was observed. In general, the different binary mixtures turned out to be antagonistic. The concentration-addition model appears to be a good tool to predict the toxicity of metal mixtures.
\end{abstract}

Key words: Concentration-addition model, synergistic ratio model, interactions, toxicity, Dahpnia obtusa, bioassay.

\section{RESUMEN}

Evaluación de la toxicidad de mezclas binarias de cobre, cinc y arsénico sobre Daphnia obtusa (Kurz, 1874) (Cladocera, Crustacea)

La protección de los ecosistemas acuáticos se basa en normas ambientales que considera las concentraciones máximas permisibles de las sustancias químicas individualmente. Sin embargo, en la realidad existen mezclas complejas en las que se producen múltiples interacciones entre los constituyentes (i.e. aditiva, sinérgica, antagónica). Entre los metales presentes en altas concentraciones en los ecosistemas lóticos afectados por la actividad minera pueden mencionarse el cobre, el zinc y el arsénico. En esta investigación se propuso determinar qué tipo de interacciones se dan entre estos elementos, para lo cual se realizaron pruebas de toxicidad aguda con mezclas binarias de Zn:As, Zn:Cu y Cu:As sobre el cladócero Daphnia obtusa. El tipo de interacción se determinó a través de los modelos concentración-adición y proporción de sinergia. Los resultados muestran que el cinc ejerce una interacción antagónica sobre el arsénico, en tanto que el arsénico ejerce un efecto sinérgico sobre el cinc. En cuanto a la interacción $\mathrm{Zn}: \mathrm{Cu}$ al incrementar la concentración del cinc en la mezcla se observó un efecto antagónico sobre el cobre. En general las diferentes mezclas binarias resultaron ser antagónicas. El modelo de concentraciónadición resulta ser una buena herramienta para predecir la toxicidad de mezclas de metales.

Palabras clave: Modelo de Concentración-adición, modelo de proporción de Sinergia, interacciones, toxicidad, Dahpnia obtusa, bioensayo. 


\section{INTRODUCCIÓN}

Los ecosistemas acuáticos reciben una gran diversidad y cantidad de compuestos químicos provenientes de múltiples actividades humanas (i.e. agrícola, industrial, minera) (Khangarot \& Rathora, 2003, Banks et al., 2005). Para proteger los organismos que habitan estos ecosistemas, se han establecido normas y/o regulaciones ambientales basadas en concentraciones totales y disueltas máximas de los elementos químicos en forma individual sin considerar el papel que juegan los factores ambientales. Así, esta aproximación no garantiza la protección de los organismos acuáticos debido a que la toxicidad de los agentes químicos es afectada por factores físicos y químicos como $\mathrm{pH}$, dureza, materia orgánica (De Schamphelaere \& Janssen, 2004). Otro factor que puede afectar la toxicidad individual de un contaminante es la presencia de otros contaminantes, debido a las interacciones que ocurren en mezcla (sinérgica, antagónica, aditiva) (Faust et al., 2003, Ren et al., 2004). En los últimos años ha habido un creciente interés por el estudio de las interacciones entre los agentes químicos, que permita predecir su impacto sobre los organismos que habitan los ecosistemas acuáticos (Gramatica et al., 2001, Barata et al., 2006).

Los metales pesados se destacan por su toxicidad sobre los organismos en los ecosistemas acuáticos (Hellawell, 1992), su efecto se relaciona con una alteración de la homeostasis de cationes como $\mathrm{Ca}^{+2}, \mathrm{Mg}^{+2}$, afectando el transporte a nivel de membrana celular (Pane et al., 2003). También afectan los mecanismos de respiración disminuyendo la tasa de respiración, además, como en el caso del cobre, este aumenta la fragilidad de la membrana lisosomal liberando enzimas hidrolíticas (Khangarot \& Rathore, 2003). Esto ha conducido a establecer normas de calidad basadas en concentraciones en forma individual de los agentes químicos en medios acuáticos.

Para predecir la toxicidad de agentes químicos se han desarrollado modelos como el Modelo del Ligando Biótico, sin embargo esta herramienta predictiva no considera la interacción entre los agentes químicos en mezcla (Niyogi $\&$ Wood, 2004). Esto hace necesario contar con herramientas predictivas de la interacción entre agentes químicos, para ello se ha propuesto el modelo de concentración-adición como una solución al problema de predecir la toxicidad de mezclas de metales. (Backhaus et al., 2004, Ren et al., 2004).

Para evaluar la toxicidad de mezclas de elementos químicos en aguas superficiales, normalmente se utilizan organismos acuáticos tales como Daphnia magna y Daphnia pulex (Gaete \& Paredes, 1996, Barata et al., 2006). Estos organismos se utilizan por su alta tasa de reproducción, fácil manipulación y sensibilidad a los contaminantes (Silva et al., 2003) La Daphnia obtusa, es una especies de cladóceros presentes en los ecosistemas acuáticos en Chile y está siendo utilizada como organismos de prueba para bioensayos de toxicidad (Álvarez, 2004, Villalobos, 2006).

Los ecosistemas acuáticos afectados por aguas de escorrentía de explotación mineras, en general presentan altos niveles de contaminación por cobre, cinc y arsénico, lo que representa un riesgo para las comunidades de organismos acuáticos que habitan dichos ecosistemas (Gaete et al., 2007). Esto plantea la necesidad de contar con información ecotoxicológica que permita conocer el tipo de interacción que ocurre entre estos metales, para una mejor predicción de sus efectos sobre los organismos acuáticos que habitan estos ecosistemas. Por ello, en esta investigación se propuso determinar el tipo de interacción entre estos agentes químicos, para lo cual se realizaron pruebas de toxicidad aguda con mezclas binarias de $\mathrm{Zn}: \mathrm{As}$, $\mathrm{Zn}: \mathrm{Cu}$ y Cu:As sobre el cladócero Daphnia obtusa.

\section{MATERIALES Y MÉTODOS}

Los ensayos de toxicidad fueron realizados con el cladócero Dahpnia obtusa (neonatos de menos de 24 horas), cultivados en el Laboratorio de Biología de la Facultad de Ciencias de la Universidad de Valparaíso. Los dáfnidos fueron mantenidos en medio de prueba de acuerdo a la norma chilena INN (1999), con pH $7.8 \pm 0.2$, dureza $120 \pm$ $25 \mathrm{mg} / \mathrm{l}$ (como $\mathrm{CaCO}_{3}$ ), OD mayor a $80 \%$ de saturación, temperatura $20 \pm 2{ }^{\circ} \mathrm{C}$, fotoperíodo $16 \mathrm{~h}$ 
Tabla 1. Concentraciones de los elementos químicos probados en los bioensayos de toxicidad con D. obtusa. Concentrations of the chemical elements tested in the toxicity bioassays with D. obtusa.

\begin{tabular}{|lr|}
\hline $\begin{array}{l}\text { Elementos } \\
\text { Químicos }\end{array}$ & Concentraciones crecientes $(\mu \mathrm{g} / \mathrm{L})^{*}$ \\
\hline Arsénico & $1-3-6-12-24-$ control \\
Cobre & $3-9-18-36-72-$ control \\
Cinc & $30-60-120-240-480-$ control \\
\hline
\end{tabular}

luz/8 h oscuridad y alimentados con un concentrado de pescado $(6.3 \mathrm{~g})$, alfalfa $(0.5 \mathrm{~g})$ levadura (2.6 g) y microalgas (Pseudokirchrneriella subcapitata) una concentración de $100000 \mathrm{cel} / \mathrm{mL}$, tres veces por semana con $3 \mathrm{~mL}$ de alimento por litro de cultivo de Daphnia obtusa, una cantidad mayor obstruye el sistema filtrador del cladócero (Silva et al., 2003). Se realizaron pruebas de toxicidad aguda, de acuerdo a la norma INN(1999), se determinó la concentración letal que provoca la mortalidad en el cincuenta porciento de los individuos a las veinticuatro horas de exposición $\left(\mathrm{CL}_{50}-24 \mathrm{~h}\right)$ y el criterio de aceptabilidad fue la sobrevivencia en los controles mayor a $90 \%$.

En los experimentos se consideraron tres metales pesados presentes en cuerpos de agua afectados por la actividad minera. Las soluciones stock fueron preparadas a partir de los siguientes reactivos de grado analítico Merck(C) $99 \%$ de pureza: $\mathrm{CuSO}_{4 *} 5 \mathrm{H}_{2} \mathrm{O}, \mathrm{ZnCl}_{2}$ y $\mathrm{As}_{2} \mathrm{O}_{3}$. El primer experimento consistió en determinar la toxicidad aguda de cada metal en forma individual. Las pruebas consistieron en cinco concentraciones para cada metal más un control (Tabla 1). En cada tratamiento se emplearon 20 individuos por concentración divididos en cuatro réplicas. Los valores de $\mathrm{CL}_{50}-24 \mathrm{~h}$ en cada una de las pruebas fueron calculados a través del análisis Probit (Finney, 1971).

El segundo experimento consistió en determinar el tipo de interacción de acuerdo a Otitoloju (2002 y 2003) para ello se determinó la toxicidad aguda de las mezclas binarias de las sales de los metales pesados $\mathrm{Zn}: \mathrm{As}$; $\mathrm{Zn}: \mathrm{Cu}$ y $\mathrm{Cu}: \mathrm{As}$, en las siguientes proporciones predeterminadas $4: 1$, $3: 2,1: 1,2: 3$, y 1:4, utilizando 5 concentraciones crecientes para cada proporción de las mezclas indicadas en la Tabla 2. El tipo de interacción se determinó de acuerdo al modelo proporción-sinergia (Hewlett \& Plackett, 1969) y concentración-adición (Anderson \& Weber, 1975). Según este modelo la proporción de sinergia (PS) se determina mediante,

$$
\mathrm{PS}=\frac{\mathrm{CL}_{50} \text { del elemento químico actuando individualmente }}{\mathrm{CL}_{50} \text { de la mezcla }}
$$

donde PS $=1$ indica aditividad, mientras que PS $>1$ indica sinergia y PS $<1$ antagonismo.

\section{Concentración-Adición (CA)}

Este modelo asume que cuando dos tóxicos actúan de modo similar siendo mezclados en cualquier proporción, ellos se sumaran para dar la respuesta observada (Otitoloju, 2002). El valor de $\mathrm{CL}_{50}$ estimada se obtiene sumando los valores de $\mathrm{CL}_{50}$ de los metales obtenido experimentalmente de forma individual según su contribución en la mezcla de acuerdo a las proporciones probadas. Luego estos valores de $\mathrm{CL}_{50}$ estimados son divididos por los valores obteni-

Tabla 2. Concentraciones crecientes para mezclas binarias probadas en bioensayos de toxicidad con D. obtusas. Increasing concentrations for the tested binary mixtures in toxicity bioassays with $\mathrm{D}$. obtusa.

\begin{tabular}{|cr|}
\hline $\begin{array}{c}\text { Proporción } \\
\text { Zn:As }\end{array}$ & Concentraciones probadas $(\mu \mathrm{g} / \mathrm{L})$ \\
\hline $4: 1$ & $5-10-30-60-120-$ control \\
$3: 2$ & $5-10-30-60-120-$ control \\
$1: 1$ & $5-10-20-40-80-$ control \\
$2: 3$ & $3-6-18-36-72-$ control \\
$1: 4$ & $2-4-8-16-32-$ control \\
\hline Proporción & \\
Zn:Cu & $5-10-30-60-120-$ control \\
\hline $4: 1$ & $5-10-30-60-120-$ control \\
$3: 2$ & $5-10-20-40-80-$ control \\
$1: 1$ & $5-10-20-40-80-$ control \\
$2: 3$ & $5-8-16-32-64-$ control \\
$1: 4$ & \\
\hline Proporción & Concentraciones probadas $(\mu \mathrm{g} / \mathrm{L})$ \\
Cu:As & $4-8-16-32-64-$ control \\
\hline $4: 1$ & $4-8-16-32-64-$ control \\
$3: 2$ & $3-6-12-24-48-$ control \\
$1: 1$ & $3-6-12-24-48-$ control \\
$2: 3$ & $2-4-8-16-32-$ control \\
$1: 4$ & \\
\hline
\end{tabular}


Tabla 3. Valores medios de $\mathrm{CL}_{50}-24 \mathrm{~h}$ para los elementos químicos evaluados individualmente con $D$. obtusa, entre paréntesis min-max. Average $C L_{50}-24 h$ values for the individually assessed chemical elements with $\mathrm{D}$. obtusa, between brackets min-max.

\begin{tabular}{|lc|}
\hline $\begin{array}{l}\text { Elementos } \\
\text { Químicos }\end{array}$ & CL50-24h $\left(95 \%\right.$ LC) $(\mu \mathrm{g} / \mathrm{L})^{*}$ \\
\hline Cobre & $15.8(7.2-28.2)$ \\
Cinc & $98.0(61.9-137.9)$ \\
Arsénico & $6.1(2.7-18.6)$ \\
\hline
\end{tabular}

dos experimentalmente de la mezcla binaria, para así determinar la clase de acción conjunta de acuerdo con la siguiente ecuación,

$$
\mathrm{CA}=\frac{\mathrm{CL}_{50} \text { estimada }}{\mathrm{CL}_{50} \text { experimental de la mezcla }}
$$

donde $\mathrm{CA}=1$ indica que el efecto es aditivo, $\mathrm{CA}>1$ que hay sinergia y que $\mathrm{CA}<1$ que hay antagonismo.

\section{RESULTADOS}

En la Tabla 3 se indican los valores de $\mathrm{CL}_{50}-24 \mathrm{~h}$ para los metales en forma individual, el metal más tóxico fue arsénico, seguido de cobre y luego el cinc. El arsénico resultó ser 16 veces más tóxico que el cinc y cerca de 3 veces más tóxico que el cobre.

La toxicidad de la mezcla Zn:As aumenta al incrementarse la proporción de arsénico con respecto al cinc (Tabla 4) excepto en la mezcla en la proporción 4:1 que resultó menos tóxica que el cinc individualmente. Por otro lado al comparar las $\mathrm{CL}_{50}-24 \mathrm{~h}$ de las mezclas con la toxicidad del arsénico actuando de forma individual, todas las proporciones de esta mezcla fueron menos tóxicas (Tabla 4).

En relación al tipo de interacción de la mezcla Zn:As, según el modelo de proporción-sinergia, para el caso del cinc todos los cocientes fueron superiores a uno, clasificándose la interacción como sinérgica, excepto en la proporción 4:1 cuyo valor de PS fue 0.9 , clasificándose como antagónica (Tabla 4). Los valores de cociente con respecto al arsénico, en todas las mezclas fueron menores a uno, indicando antagonismo para el arsénico mezclado con cinc (Tabla 4).

Según el modelo concentración-adición para la mezcla $\mathrm{Zn}$ :As, en ella las proporciones 3:2, 1:1 y 4:1 la toxicidad experimental fue similar a la toxicidad estimada (Tabla 5), en las otras

Tabla 4. Valores medios de $\mathrm{CL}_{50}-24 \mathrm{~h}$ para metales individuales y en mezclas binarias probadas en $D$. obtusa. Average $C L_{50}-24 \mathrm{~h}$ values of metals tested individually and in binary mixtures on $\mathrm{D}$. obtusa.

\begin{tabular}{|cccc|}
\hline Mezcla & CL50- 24h $(95 \%$ LC) $(\mu \mathrm{g} / \mathrm{L})$ & PS1 & PS2 \\
\hline Zn-As & $104.7(65.9-495,2)$ & Zn & 0.05 \\
$4: 1$ & $60.7(29,0-180,6)$ & 1.61 & 0.10 \\
$3: 2$ & $46.1(19,8-455,5)$ & 2.09 & 0.13 \\
$1: 1$ & $22.0(11.6-36.7)$ & 4.44 & 0.27 \\
$1: 3$ & $07.7(3.8-13.7)$ & 7.11 & Cu \\
& & Zn & 0.16 \\
Zn-Cu & $93.8(56.0-308.1)$ & 1.04 & 0.22 \\
$4: 1$ & $69.1(40.7-129.5)$ & 1.41 & 0.29 \\
$1: 1$ & $56.4(34.8-123.8)$ & 1.83 & 0.35 \\
$2: 3$ & $44.7(29.3-75.6)$ & 2.19 & 0.19 \\
$1: 4$ & $32.0(22.4-45.6)$ & 3.05 & Cu \\
\hline Cu-As & & 0.70 & 0.27 \\
$4: 1$ & $22.5(15.1-30.9)$ & 0.72 & 0.28 \\
$1: 1$ & $21.6(14.8-29.0)$ & 0.87 & 0.33 \\
$2: 3$ & $18.1(10.2-33.3)$ & 0.69 & 0.26 \\
& $22.8(14.5-30.2)$ & 1.36 & 0.52 \\
\hline
\end{tabular}


Tabla 5. Valores de $\mathrm{CL}_{50}-24 \mathrm{~h}$ experimentales y $\mathrm{CL}_{50}-24 \mathrm{~h}$ estimadas de las mezclas binarias. ${ }^{*}$ Diferencia significativa ( $\left.p<0.05\right)$, entre la toxicidad experimental y estimada, mediante la prueba de T. Values of experimental $C L_{50}-24 \mathrm{~h}$ and estimated $C L_{50}-24 h$ of the binary mixtures. ${ }^{*}$ Significant difference $(\mathrm{p}<0.05)$ between experimental and estimated toxicity with a $T$ test.

\begin{tabular}{|cccc|}
\hline Mezcla de metales & CL50-24h estimada (LC 95\%) (ug/L) & CL50-24h experimental (LC 95\%) (ug/L) & CA \\
\hline Zn-As & $79.6(50.0-114.0)$ & & \\
$4: 1$ & $61.21(38.2-90.2)$ & $104.7(65.9-495.2)$ & $0.76^{*}$ \\
$3: 2$ & $52.0(31.3-88.1)$ & $60.7(29.0-180.6)$ & 1.01 \\
$1: 1$ & $42.8(26.4-66.3)$ & $46.1(18.8-455.5)$ & $1.13^{*}$ \\
$2: 3$ & $24.4(15.9-47.0)$ & $22.0(11.6-36.7)$ & $3.15^{*}$ \\
$1: 4$ & & $7.7(3.9-13.7)$ & \\
\hline $\mathrm{Zn}-\mathrm{Cu}$ & $81.5(50.9-116.0)$ & & $0.86^{*}$ \\
$4: 1$ & $65.1(40.0-94.0)$ & $93.8(56.0-308.1)$ & $0.94^{*}$ \\
$3: 2$ & $56.9(34.5-83.0)$ & $69.1(40.7-129.5)$ & 1.00 \\
$1: 1$ & $48.6(29.0-72.1)$ & $46.4(34.8-123.8)$ & 1.00 \\
$2: 3$ & $32.2(18.1-50.1)$ & $32.0(22.4-45.6)$ & \\
$1: 4$ & & & \\
& & $22.5(15.1-30.9)$ & $0.62^{*}$ \\
$4: 1$ & $13.8(6.3-26.2)$ & $21.67(14.8-29.0)$ & $0.55^{*}$ \\
$3: 2$ & $11.9(5.4-24.3)$ & $18.1(10.2-33.3)$ & $0.60^{*}$ \\
$1: 1$ & $10.9(4.98-33.2)$ & $22.8(14.5-30.2)$ & $0.44^{*}$ \\
$1: 3$ & $10.0(4.53-22.4)$ & $11.6(6.5-20.2)$ & $0.69^{*}$ \\
\hline
\end{tabular}

mezclas la toxicidad experimental fue mayor que la estimada. En cuanto a los cocientes concentración adición (CA) sólo la proporción 4:1 presenta interacción del tipo antagónica con un cociente menor a uno, mientras que las otras proporciones mostraron sinergismo, presentando cocientes con valores mayores a uno. La mezcla en la proporción 1:4 de Zn:As, presentó el cociente más alto de sinergismo de 3.15 (Tabla 5). Se observó una gran similitud entre la toxicidad estimada según el modelo concentración-adición y la toxicidad experimental.

La Tabla 4 indica que la mezcla $\mathrm{Zn}: \mathrm{Cu}$, la toxicidad se incrementa al aumentar la proporción del cobre en ella. Todas las proporciones de la mezcla fueron más tóxicas con respecto a la toxicidad del cinc en forma individual, la proporción 4:1 fue la que presentó menor toxicidad siendo similar a la toxicidad del cinc individualmente. Al comparar la toxicidad de esta mezcla con la toxicidad del cobre actuando individualmente, todas las mezclas en las distintas proporciones fueron menos tóxicas. Con relación al tipo de interacción según el modelo proporción-sinergia, el cociente, para el caso del cinc fue mayor a uno, clasificándose la interacción como sinérgica, siendo la mezcla en la proporción 1:4 la más alta con un cociente de 3.05. Respecto a los cocientes del cobre, todos fueron menores a uno, clasificándose como antagónica la mezcla para este metal (Tabla 4). La toxicidad estimada según el modelo concentración-adición y la toxicidad experimental fueron similares (Tabla 5). En cuanto a los cocientes de concentración-adición para la mezcla $\mathrm{Zn}: \mathrm{Cu}$, se observa que las proporciones 4:1 y 3:2 la interacción es antagónica (Tabla 5), en tanto que las proporciones $1: 1$ y $1: 4$, se clasifican como aditivas. Sólo la proporción $2: 3$ resultó ser sinérgica con un cociente de 1.08 , pero no significativamente (Tabla 5).

Con relación a la mezcla $\mathrm{Cu}$ :As la proporción más tóxica fue 1:4, en tanto que la menos tóxica fue la mezcla en la proporción 2:3 (Tabla 4). Las proporciones 4:1, 3:2, 1:1 y 2:3 fueron menos tóxicas que el cobre actuando individualmente, clasificándose como interacción de tipo antagónica (tabla 4). Similares resultados se encontraron en relación al arsénico, siendo todas las proporciones clasificadas como interacción de tipo antagónica (Tabla 4).

La Tabla 5 indica que para la mezcla $\mathrm{Cu}: \mathrm{As}$, la toxicidad experimental fue menor que la toxici- 
dad estimada significativamente, según el modelo concentración adición. En cuanto a los cocientes obtenidos mediante el modelo de concentraciónadición, estos fluctuaron entre 0.44 y 0.69 siendo la proporción 2:3 la de menor toxicidad y la más tóxica la proporción 1:4, clasificándose todas las interacciones como de tipo antagónica (Tabla 5).

\section{DISCUSIÓN}

Las especies químicas del arsénico pueden corresponder a $\mathrm{As}^{\mathrm{V}}$ y $\mathrm{As}^{\mathrm{III}}$, siendo esta última la más tóxica para los organismos acuáticos. La toxicidad aguda $\left(\mathrm{CL}_{50}-24 \mathrm{~h}\right)$ del As ${ }^{\mathrm{III}}$ sobre Daphnia pulex y Daphnia magna fluctúa entre 1.0 y $5.2 \mu \mathrm{g} / \mathrm{L}$, (Jureswicz \& Buikema, 1980), en este estudio la toxicidad del arsénico fue levemente mayor con una CL50-24h de $6.1 \mu \mathrm{g} / \mathrm{L}$. En cuanto a la toxicidad aguda del cobre $(15.8 \mu \mathrm{g} /)$, estuvo dentro de los rangos descritos para invertebrados de agua dulce, la cual fluctúa entre 5.4 y $53 \mu \mathrm{g} / \mathrm{L}$ (Mount \& Norberg, 1984; Moore, 1991; Silva et al., 2003; Barata et al., 2006). La toxicidad del zinc fue similar a lo descrito por Zou $\& \mathrm{Bu}$ (1994) quienes señalan que la $\mathrm{CL}_{50}-24 \mathrm{~h}$ fluctúa entre 81.3 y $327.3 \mu \mathrm{g} / \mathrm{L}$, en el presente estudio el valor medio de la $\mathrm{CL}_{50}-24 \mathrm{~h}$ del zinc como $\mathrm{ZnCl}_{2}$, fue $98 \mu \mathrm{g} / \mathrm{L}$.

En la mezcla de Zn:As, el modelo de proporción-sinergia para la proporción 4:1 mostró una interacción del tipo antagónico, lo cual se atribuye a la alta proporción de $\mathrm{Zn}$, aunque este metal es 16 veces menos tóxico que el arsénico, el cual se encontró conformando el $20 \%$ de la mezcla. En las proporciones $3: 2 ; 1: 1 ; 2: 3$ y $1: 4$, la toxicidad fue más alta debido al aumento del arsénico en la mezcla. Al analizar el modelo proporción-sinergia respecto al zinc, las mezclas fueron sinérgicas, en particular la mezcla en la proporción 1:4, esto debido a la alta cantidad de arsénico en la mezcla. En cuanto al modelo proporción-sinergia respecto al arsénico, estas fueron antagónicas, siendo la mezcla 4:1 la mezcla más antagónica. Esta interacción depende de la concentración de los metales en mezcla lo que es similar a los descrito por Fargasova (2001) en mezclas de $\mathrm{Zn}$ :Cd sobre estados larvales de $\mathrm{Chi}$ - romonus plumosus. Una posible explicación de este antagonismo podría estar relacionado con la competencia entre estos agentes químicos por los sitios de acción en la interfase biológica (Bryan \& Gibbs, 1983).

Al aplicar el modelo concentración-adición a la mezcla Zn:As, la proporción 4:1 fue clasificada como antagónica, en tanto las otras proporciones como sinérgicas, coincidiendo con el modelo de proporción-sinergia. Enserink et al., (1991), quien trabajando con mezclas equitóxicas de zinc y arsénico en presencia de otros metales con Daphnia magna, las clasificó estas interacciones como aditivas.

En la mezcla $\mathrm{Zn}: \mathrm{Cu}$, el cobre ejerce un efecto sinérgico sobre el zinc al aumentar su proporción en la mezcla, en tanto que el zinc provoca un efecto antagónico cuando está en alto porcentaje en la mezcla. Esto es similar a lo observado por Parrott \& Sprague (1993), quienes encontraron una mayor alteración de la estructura del ADN y proteínas en Pimephales promelas provocadas por el cobre y zinc individualmente que cuando estos metales estaban en mezcla. Por otra parte, Utgikar et al. (2004), describen una interacción sinérgica entre el cinc y el cobre sobre $\mathrm{Vi}$ brio fischer que es atribuible al cobre. La interacción antagónica podría estar relacionada con la electronegatividad de los metales en mezcla como también el diámetro de los iones que interactúan con similares sitios activos en los organismos (Fargasova, 2001). Sin embargo, Khangarot et al. (1982), observaron un efecto sinérgico entre estos metales en igual proporción, que este estudio, pero sobre el caracol de agua dulce Lymnaea acuminata. Esta diferencia podría ser atribuible a la diferencia de sensibilidad y características fisiológicas entre los organismos.

En la mezcla $\mathrm{Cu}$ :As los valores de proporción sinergia de cobre en presencia de arsénico, fueron menores a uno, lo que indica que existe un antagonismo en la mezcla, excepto para la mezcla 1:4, en cuanto a lo indicado por el modelo de proporción sinergia para el arsénico en presencia de cobre, todas las proporciones fueron menores a uno, indicando una interacción de tipo antagónica. El modelo de concentración-adición indica que esta mezcla en todas sus proporciones 
se clasifica como de antagonismo. La toxicidad del arsénico en los organismos acuáticos depende de la forma química en la que se encuentre, las formas orgánicas del arsénico la mayoría de las veces no son muy toxicas, mientras que sus derivados inorgánicos son más tóxicos para los organismos acuáticos (Sproal, 2002). Estudios realizados por Genter \& Lehman (2000), al comparar la comunidad de diatomeas entre una zona localizada aguas arriba de una actividad minera, donde la concentración de arsénico era de $6 \mu \mathrm{g} / \mathrm{L}$, con la zona aguas abajo de la mina, donde las concentraciones de cobre era de $97 \mu \mathrm{g} / \mathrm{L}$, encontraron un disminución de la riqueza de diatomeas, atribuible al efecto sinérgico del cobre con el arsénico. Esta diferencia con nuestro estudio podría ser debido a las especies utilizadas correspondientes a productores primarios que presentarían distinta sensibilidad al efecto toxico de cobre y arsénico.

En este estudio la predicción de la toxicidad de las mezclas mediante el modelo concentración-adición fueron en general similares a las observadas en las diferentes proporciones. En tanto que para la mezcla $\mathrm{Cu}$ :As el modelo predijo una mayor toxicidad lo cual es similar a lo descrito por Faust et al. (2003) y Backhaus et al (2004). El efecto antagónico del cinc sobre el arsénico y el cobre cuando está en altas concentraciones podría ser beneficioso para la protección de los organismos acuáticos, similar situación ocurre entre cobre y arsénico. En el trabajo de Barata et al., (2006) se encontró una interacción entre cobre y cadmio de tipo menos que aditiva, lo cual es similar en el presente estudio, especialmente en la interacción $\mathrm{As}: \mathrm{Cu}$, estos autores señalan como posible explicación, que estos metales inducen la producción metalotioneinas y como consecuencia es esperable que toxicidad combinada sea antagónica.

Finalmente, el concepto de concentración adición es fácil de aplicar pero en algunos casos podría sobreestimar la toxicidad de mezclas. Sin embargo, desde la perspectiva regulatoria esta sobreestimación podría ser beneficiosa para la protección de los organismos acuáticos. Este modelo puede ser aplicable como herramienta de carácter predictivo para la protección de los ecosistemas acuáticos.

\section{AGRADECIMIENTOS}

Este trabajo fue financiado por la Dirección de Investigación de la Universidad de Valparaíso a través del Proyecto Dipuv 06/2003.

\section{BIBLIOGRAFÍA}

ÁLVAREZ, A. S. 2004. Evaluación de toxicidad de las aguas superficiales afectada por la actividad minera en la cuenca del Aconcagua (Valparaíso, Chile). Tesis de para optar al título de Ingeniero Ambiental, Facultad de Ciencias. Universidad de Valparaíso. 82 pp.

ANDERSON, P. D. \& L. J. WEBER. 1975. The toxicity to aquatic population of mixtures containing certain heavy metals. In: Proceeding of the International Conference on heavy Metal in The enviroment, Institute of Enviromental Studies, University of Toronto. 933-953 pp.

BACKHAUS, T., A. ARRHENIOUS \& H. BLANCK. 2004. Toxicity of dissimilarly acting substances to natural algal communities: predictive power and limitations of independent action and concentration addition. Environ. Sci. Technol., 38: 6363-6370.

BANKS, K., P. TURNER, S. WOOD \& C. MATTHEWS. 2005. Increased toxicity to Ceriodaphnia dubia in mixtures of atrazine and diazinon at environmentally ealistic concentrations. Ecotoxicology and Environmental Safety, 60: 28-36.

BARATA C., D. J. BAIRD, A. J. NOGUEIRA, A. M. SOARES \& M. C. RIVA. 2006. Toxicity of binary mixtures of metals and pyrethroid insecticides of Dapnia magna Straus. Implications for mult-substance risks assessment. Aquatic Toxicology, 78: 1-14.

BEISINGER K. E., G. M. CHRISTENSEN \& J. T. FIANT.1986. Effects of metal salt mixtures on Daphnia magna reproduction. Ecotoxicology and Environmental Safety, 11: 9-4.

BRYAN, G. W., \& P. E. GIBBS. 1983. Heavy metals in the Fal estuary, Cornwall; a study of long-term contamination by mining waste and its effects on estuarine organisms. Estuaries, 10(3): 208-219.

BUHL, K. 1998. Toxicity Of proposed water quality criteria-based mixtures of 11 inorganics to Ceriodaphnia dubia and Fathead minnow. Final report 
U.S. Geological Survey Environmental and Contaminants research Center, ecotoxicology Research station. Yankton, South Dakota. 150 pp.

DE SCHAMPHELAERE, C. \& C. JANSSEN. 2004. Effects of dissolved organic carbon concentration and source, $\mathrm{pH}$, and water hardness on chronic toxicity of copper to Daphnia magna. Environmental Toxicology and Chemistry, 23(5): 1115-1122.

ENSERINK, E. L., J. L. MAAS-DIEPEVEN \& C. J. VAN-LEEUWEN.1991. Combined effects of metals; an ecotoxicological evaluation. Water Res., 25(5): 679-687.

FARGASOVA, A. 2001. Winter Third- to FourthInstar Larvae of Chironomus plumosus as Bioassay Tools for Assessment of Acute Toxicity of Metals and Their Binary Combinations. Ecotoxicology and Environmental Safety, 48: 1-5.

FAUST, A M., R. ALTENBURGER, T. BACKHAUS, H. BLANCK, W. BOEDEKER, P. GRAMATICA, V. HAMER, M. SCHOLZE, M. VIGHI \& L. GRIMME. 2003. Joint algal toxicity of 16 dissimilarly acting chemicals is predictable by the concept of independent action. Aquatic Toxicology, 63: 43-63.

GAETE, H., F. ARANGUIZ, G. CIENFUEGOS y M. TEJOS. 2007. Toxicidad y metales pesados en el río Aconcagua, Química Nova, 30(4): 885-891.

GAETE, H. y K. PAREDES. 1996. Toxicidad de mezclas de contaminantes químicos sobre el Cladocero Daphnia magna. Rev. Int. Contam. Ambient., 12(1): 23-28.

GENTER, R. B. \& R. M. LEHMAN. 2000. Metal toxicity inferred from algal population density, heterotrophic substrate use, and fatty acid profile in a small stream. Enviromental Toxicology and Chemistry, 19(4): 869-878.

GRAMATICA P., M. VIGHI, F. CONSOLARO, R. TODESCHINI, A. FINIZIO \& M. FAUST. 2001. QSAR approach for the selection of congeneric compounds with a similar toxicological mode of action. Chemosphere, 42: 873-883.

FINNEY, D. J. 1971. Probit Analysis, $3^{\text {rd }}$ ed. Cambridge University Press, London. 333 pp.

HELLAWELL, J. M. 1992. Biological indicators of freshwater pollution and environmental management. Pollution Monitoring Series. Ed. Elsevier Applied Science. USA. 546 pp.

HEWLETT, P. S. \& R. L. PLACKETT. 1969. A unified theory for quantal responses to mixtures of drugs. Non-interactive action. Biometrics, 15: 591610.
INN. 1999. Bioensayo de toxicidad aguda mediante la determinación de la inhibición de la movilidad de Daphnia magna o Daphnia pulex (Crustacea, Cladocera). Norma Chilena NCh2083/1999. Instituto Nacional de Normalización, Chile. 10 pp.

JURESWICZ, S. \& A. L. BUIKEMA. 1980. Effects of arsenate on algae, daphnia and mosquito fish, Virginia. J. Sci., 31:124. Cited in EPA. 1985.

KHANGAROT, B. S., S. MATHUR \& V. S. DURVE. 1982. Comparative toxicity of heavy metals and interaction of metal on freshwater pulmonate snail, Lymnaea acuminata. L. Acta Hydrochim. Hydrobiol., 10(4): 367-375.

KHANGAROT, B. \& R. RATHORE. 2003. Effects of Copper on Respiration, Reproduction, and Some Biochemical Parameters of Water Flea Daphnia magna Straus. Bull. Environ. Contam. Toxicol., 70: 112-117.

MOORE JAMES, W. 1990. Inorganic contaminants of surface water. Springer Series on Environmental Management Vol II. Ed. Springer-Verlag. $334 \mathrm{pp}$.

MOUNT, D. I. \& T. J. NORNBERG. 1984. A seven day life-cycle cladoceran toxicity tests. Environt. Toxicol. Chem, 3:425. Cited in EPA, 1985.

NIYOGI, S. \& C. WOOD. 2004. Biotic Ligand Model, a Flexible Tool for Developing Site-Specific Water Quality Guidelines for Metals. Environmental Science and Technology, 38(23): 6177-6192.

OTITOLOJU, A. A. 2002. Evaluation of the jointaction toxicity of binary mixtures of heavy metals against the mangrove periwinkle Tympanotonus fuscatus var radula (L.). Ecotoxicol. Environ. Saf, 53: 404-415.

OTITOLOJU, A. A. 2003. Relevance of joint action toxicity evaluations in setting realistic environmental safe limits of heavy metals. Journal of Environmental Management, 67: 121-128.

PANE, E. F. C., J. C. SMITH, C. MCGEER \& C. WOOD. 2003. Mechanisms of Acute and Chronic Waterborne Nickel Toxicity in the Freshwater Cladoceran, Daphnia magna. Environ. Sci. Technol., 37: 4382-4389.

PARROT, J. L. \& J. B. SPRAGUE. 1993. Patterns in toxicity of sublethal mixtures of metal and organic chemicals determined by microtox and by DNA, RNA and protein content of fathead minnows Pimephales promelas. Can. J Fish. Aquat. Sci., 50(10): 2245-2253. 
RAYNER-CANHAN, G. 2000. Química inorgánica descriptiva. $2^{\text {a }}$ ed. Prentice Hall, Pearson Educación. México. 345 pp.

REN, S., R. MEE \& P. FRYMIER. 2004. Using factorial experiments to study the toxicity of metal mixtures. Ecotoxicology and Environmental Safety, 59: 38-43.

SPROAL, R., N. J. TUROCZY \& F. STAGNITT. 2002. Chemical and physical speciation of arsenic in a small pond receiving gold mine waste effluent. Ecotoxicology and Environmental Safety, 53(3): 370-375.

SILVA, J., G. TORREJON, E. BAY-SCHMITH y A. LARRAIN. 2003. Calibración de bioensayo de to- xicidad aguda con Daphnia pulex (crustacea, Cladocera) usando tóxico de referencia. Gayana, 67(1): 87-96.

UTGIKAR, V., N. CHAUDHARY, A. KOENIGER, H. TABAK, J. HAINES \& R. GOVIND. 2004. Toxicity of metals and metal mixtures: analysis of concentration and time dependence for zinc and copper. Water Research, 38: 3651-3658.

VILLALOBOS, L. 2006. Estado de conocimiento de los crustáceos zooplanctónicos dulceacuícolas de Chile. Gayana (Concepción), 70: 31-39.

ZOU, E. B. S. 1994. Acute toxicity of copper, cadmium and zinc to the water flea, Moina irrasa (Cladocera). Bull. Environ. Contam. Toxicol., 52: 742-748. 